\title{
আর্কেড ফাউন্ডেশন
}

ইমপ্যাক্ট অফ কোভিড-১৯ অন ওল্ডার এ্যাডাল্টস ইন বাংলাদেশ

\section{প্রশ্নমালা}

খানার আইডিঃ

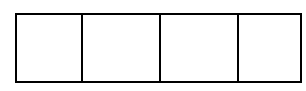

তারিখ:

উত্তরদাতার নাম:

বাবার নাম:

গ্রাম/ক্যাম্প:

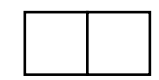

ইউনিয়ন:

উপজেলা:

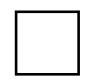

মোবাইলঃ

\begin{tabular}{|c|c|c|}
\hline দিন & মাস & বছর \\
\hline & & \\
\hline
\end{tabular}

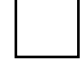

\section{A. উত্তরদাতার আর্থ-সামাজিক অবস্থা}

\begin{tabular}{|c|c|c|c|c|}
\hline $\begin{array}{l}\text { क्र. } \\
\text { নi }\end{array}$ & প্রশ্নাবলী & উত্তর & $\begin{array}{l}\text { কো } \\
ড\end{array}$ & স্কীপ \\
\hline 1. & আপনার বর্তমান বয়স কত? & (বছর) & & \\
\hline 2. & উত্তরদাতার লিঙ্গ: & $\begin{array}{l}\text { পুরুষ } \\
\text { মহিলা }\end{array}$ & $\begin{array}{l}1 \\
2\end{array}$ & \\
\hline 3. & আপনার বর্তমান বৈবাহিক অবস্থা কি? & $\begin{array}{l}\text { বিবাহিত } \\
\text { বিধবা, বিপত্নীক } \\
\text { বিবাহ বিচ্ছদ } \\
\text { আলাদা } \\
\text { আবিবাহিত }\end{array}$ & $\begin{array}{l}1 \\
2 \\
3 \\
4 \\
5\end{array}$ & \\
\hline 4. & থাকার ব্যবস্থা / কার সঙ্গে বসবাস করেন? & $\begin{array}{l}\text { একা } \\
\text { স্ত্রী } \\
\text { পুত্র } \\
\text { কন্যা } \\
\text { অন্যান্য (উল্লেখ করুন): }\end{array}$ & $\begin{array}{c}1 \\
2 \\
3 \\
4 \\
-96\end{array}$ & \\
\hline 5. & $\begin{array}{l}\text { আপনি কে আপনার জীবনযাত্রা নির্বাহের জন্য } \\
\text { আপনার পরিবারের উপর নির্ভরশীল? }\end{array}$ & $\begin{array}{l}\text { श्याँ } \\
\text { ना }\end{array}$ & $\begin{array}{l}1 \\
0\end{array}$ & \\
\hline 6. & পড়তে বা লিখতে পারেন? & $\begin{array}{l}\text { श्राँ } \\
\text { ना }\end{array}$ & $\begin{array}{l}1 \\
0\end{array}$ & \\
\hline 7. & $\begin{array}{l}\text { কোভিড-১৯ এর তথ্য আপনি কোন মাধ্যম } \\
\text { থেকে পান? } \\
\text { (একাধিক উত্তর প্রযোজ্য) }\end{array}$ & $\begin{array}{l}\text { টিভি } \\
\text { রেডিও } \\
\text { মোবাইল } \\
\text { ইন্টারনেট } \\
\text { সামাজিক মাধ্যম } \\
\text { কোনটাই না }\end{array}$ & $\begin{array}{l}1 \\
2 \\
3 \\
4 \\
5 \\
0\end{array}$ & \\
\hline 8. & পরিবারের সদস্য সংখ্যা & (নম্বর) & & \\
\hline
\end{tabular}




\section{B. নন-কমিউনিকেবল ক্রোনিক অবস্থা}

\begin{tabular}{|c|c|c|c|}
\hline & & $\begin{array}{l}\text { ১.আপনি বর্তমানে কোনও } \\
\text { দীর্ঘস্থায়ী রোগে ভুগছেন? } \\
\text { [এনুমেরেটর: প্রতিটি রোগের জন্য } \\
\text { আলাদাভাবে জিজ্ঞাসা করুন] } \\
\text { কোড: } \\
\text { ১. হ্যাঁ } \\
\text { ২. না } \\
\text { ৩. জানি না }\end{array}$ & $\begin{array}{l}\text { ২. আপনি কি এর } \\
\text { জন্য ওষুধ খাচ্ছেন? } \\
\text { কোড: } \\
\text { ১. হ্যাঁ } \\
\text { ২. না } \\
\text { ৩. জানি না }\end{array}$ \\
\hline 1. & বাত / আর্থরাইটিস & & \\
\hline 2. & উচ্চ রক্তচাপ & & \\
\hline 3. & হৃদরোগ & & \\
\hline 4. & স্ট্রোক & & \\
\hline 5. & উচ্চ মোট কোলেস্টেরল & & \\
\hline 6. & ডায়াবেটিস & & \\
\hline 7. & দীর্ঘস্থায়ী ফুসফুসের রোগ & & \\
\hline 8. & দীর্ঘস্থায়ী কিডনি রোগ & & \\
\hline 9. & ক্যান্সার & & \\
\hline 10. & অন্যান্য অসংক্রামক রোগ & & \\
\hline 11. & ছানি & & \\
\hline 12. & অন্যান্য দৃষ্টিসংক্রান্ত সমস্যা & & \\
\hline 13. & শ্রাবণ সমস্যা & & \\
\hline 14. & $\begin{array}{l}\text { অন্যান্য অসংক্রামক রোগ } \\
\text { (অনুগ্রহ করে উল্লেখ করুন) }\end{array}$ & & \\
\hline
\end{tabular}

\section{C. কোভিড-১৯ সম্পর্কিত তথ্য}

\begin{tabular}{|l|l|l|}
\hline 1. & $\begin{array}{l}\text { কোভিড-১৯ মহামারী সম্পর্কে আপনি কি } \\
\text { উদ্বিগ্ন/ চিন্তিত? }\end{array}$ & $\begin{array}{l}\text { ১. তেমন নয় } \\
\text { ২. কখনও কখনও/প্রায়ই }\end{array}$ \\
\hline 2. & $\begin{array}{l}\text { কোভিড-১৯ মহামারী নিয়ে আপনি কি অস্বস্তি } \\
\text { বোধ করছেন? }\end{array}$ & $\begin{array}{l}\text { ১. তেমন নয় } \\
\text { ২. কখনও কখনও/প্রায়ই }\end{array}$ \\
\hline 3. & $\begin{array}{l}\text { কোভিড-১৯ মহামারীতে আপনি কি একাকীত্ব } \\
\text { বোধ করছেন? }\end{array}$ & $\begin{array}{l}\text { ১. তেমন নয় } \\
\text { ২. কখনও কখনও/প্রায়ই }\end{array}$ \\
\hline 4. & $\begin{array}{l}\text { প্রকোপ শুরুর আগের মাসগুলির তুলনায়, আপনার } \\
\text { ঘনিষ্ঠ বন্ধুবান্ধব এবং পরিবারের সাথে আপনার } \\
\text { যোগাযোগ কীভাবে পরিবর্তিত হয়েছে? }\end{array}$ & $\begin{array}{l}\text { ১. আগের মতই আছে } \\
\text { ২. আগের থেকে কগে গেছে }\end{array}$ \\
\hline
\end{tabular}




\begin{tabular}{|l|l|l|}
\hline 5. & $\begin{array}{l}\text { কোভিড-১৯ মহামারীতে আপনি কি খাবার } \\
\text { সংগ্রহ করতে সমস্যায় পড়েছেন? }\end{array}$ & $\begin{array}{l}\text { ১. সমস্যা হয়নি } \\
\text { ২. সমস্যা হয়ছে }\end{array}$ \\
\hline 6. & $\begin{array}{l}\text { কোভিড-১৯ মহামারীতে আপনি কি ঔষধ সংগ্রহ } \\
\text { করতে সমস্যায় পড়েছেন? }\end{array}$ & $\begin{array}{l}\text { ১. সমস্যা হয়নি } \\
\text { ২. সমস্যা হয়ছে }\end{array}$ \\
\hline 7. & $\begin{array}{l}\text { কোভিড-১৯ মহামারীতে আপনি কি আপনার } \\
\text { নিয়মিত চিকিৎসা পেতে সমস্যায় পড়েছেন? }\end{array}$ & $\begin{array}{l}\text { ১. সমস্যা হয়নি } \\
\text { ২. সমস্যা হয়ছে }\end{array}$ \\
\hline 8. & $\begin{array}{l}\text { আপনি কি মনে করেন ওল্ডার এ্যাডাল্টসদের } \\
\text { কোভিড-১৯ এ সংক্রামিত হওয়ার সস্ভাবনা } \\
\text { সবথেকে বেশি? }\end{array}$ & $\begin{array}{l}\text { ১. হ্যাঁ } \\
\text { ২. না }\end{array}$ \\
\hline
\end{tabular}

\section{D. কোভিড-১৯ সম্পর্কিত ভয়ের স্কেল}

\begin{tabular}{|c|c|c|}
\hline 1. & আমি করোনাভাইরাস-১৯ নিয়ে খুব ভীত & $\begin{array}{l}\text { ১. দৃঢ़ভাবে অসম্মত } \\
\text { ২. অসম্মত } \\
\text { ৩. অসম্মতও না সম্মতও না } \\
\text { ৪. সম্মত } \\
\text { ৫. দৃঢ়ভাবে সম্মত }\end{array}$ \\
\hline 2. & $\begin{array}{l}\text { আমি করোনাভাইরাস-১৯ সম্পর্কে ভাবতে অস্বস্তি } \\
\text { বোধ করি }\end{array}$ & $\begin{array}{l}\text { ১. দৃঢ़ভাবে অসম্মত } \\
\text { ২. অসম্মত } \\
\text { ৩. অসম্মতও না সম্মতও না } \\
\text { ৪. সম্মত } \\
\text { ৫. দৃঢ়ভাবে সম্মত }\end{array}$ \\
\hline 3. & $\begin{array}{l}\text { করোনাভাইরাস-১৯ সম্পর্কে ভাবলে আমার হাত } \\
\text { ঘেরে ওঠে }\end{array}$ & $\begin{array}{l}\text { ১. দৃঢ़ভাবে অসম্মত } \\
\text { ২. অসম্মত } \\
\text { ৩. অসম্মতও না সম্মতও না } \\
\text { ৪. সম্মত } \\
\text { ৫. দৃঢ়ভাবে সম্মত }\end{array}$ \\
\hline 4. & $\begin{array}{l}\text { আমি করোনাভাইরাস-১৯ এর কারনে আমার জীবন } \\
\text { হারানোর ভয় পাচ্ছি }\end{array}$ & $\begin{array}{l}\text { ১. দৃঢ़ভাবে অসম্মত } \\
\text { ২. অসম্মত } \\
\text { ৩. অসম্মতও না সম্মতও না } \\
\text { ৪. সমমমমত } \\
\text { ৫. দৃঢ়ভাবে সম্মত }\end{array}$ \\
\hline 5. & $\begin{array}{l}\text { সোশ্যাল মিডিয়াতে করোনাভাইরাস-১৯ সম্পর্কিত } \\
\text { খবর বা গল্পগুলো দেখে আমি উদ্বিগ্ন হয়ে পরি }\end{array}$ & $\begin{array}{l}\text { ১. দৃত़ভাবে অসম্মত } \\
\text { ২. অসম্মত } \\
\text { ৩. অসম্মতও না সম্মতও না } \\
\text { ৪. সম্মত } \\
\text { ৫. দৃঢ়ভাবে সম্মত }\end{array}$ \\
\hline 6. & $\begin{array}{l}\text { করোনাভাইরাস-১৯ এ আক্রান্ত হওয়ার ভয়ে আমি } \\
\text { ঘুমাতে পারিনা }\end{array}$ & $\begin{array}{l}\text { ১. দৃঢ়ভাবে অসম্মত } \\
\text { ২. অসম্মত } \\
\text { ৩. অসম্মতও না সম্মতও না } \\
\text { ৪. সম্মত }\end{array}$ \\
\hline
\end{tabular}




\begin{tabular}{|l|l|l|}
\hline & & ৫. দৃढ़ভাবে সম্মত \\
\hline 7. & করোনাভাইরাস-১৯ এ আক্রান্ত হওয়ার ভয়ে আমার & ১. দৃঢ़ভাবে অসম্মত \\
& বুক ধরফর করে & ২. অসম্মত \\
& & ৩. অসম্মতও না সম্মতও না \\
& & 8. সম্মত \\
& & ৫. দৃढ़ভাবে সম্মত \\
\hline
\end{tabular}

ধন্যবাদ! 